\title{
Los saberes y competencias docentes en educación a distancia y digital. Una reflexión para la formación
}

\section{(Teacher knowledge and skills in digital and distance education. A reflection for training)}

\author{
Lorenzo García Aretio \\ UNED (España)
}

DOI: $\underline{\text { http://dx.doi.org/10.5944/ried.23.2.26540 }}$

\section{Cómo referenciar este artículo:}

García Aretio, L. (2020). Los saberes y competencias docentes en educación a distancia y digital. Una reflexión para la formación. RIED. Revista Iberoamericana de Educación a Distancia, 23(2), pp. 09-30. doi: http://dx.doi.org/10.5944/ ried.23.2.26540

\section{Resumen}

Se pretende articular el estudio y la investigación científica, con el fin de generar teorías consistentes con la práctica educativa y los procedimientos técnicos puestos en acción para dotar de mayor consistencia, calidad y, por tanto, credibilidad a los formatos educativos no presenciales. Así, abordamos en este trabajo una breve delimitación sobrelos diferentes ámbitos del conocimiento y de los saberes en el campo educativo para posteriormente, buscando esa relación, proponer los compromisos y las competencias que un docente de esta modalidad, preferentemente de nivel educativo superior, debería integrar en su corpus de conocimientos profesionales. En consecuencia, se describen los cuatro ámbitos más destacados del saber en educación: científico, teórico, técnico y práctico y las intersecciones entre ellos, para llegar a los compromisos y competencias de un docente, preferentemente universitario, que labora en formatos educativos no presenciales. Concluimos con que estos compromisos serían de carácter: profesional-laboral, ético, formativo, colaborativo y de gestión. Y en ese marco se englobarían los ámbitos de competencias docentes que deberían abarcar cuatro áreas: a) competencia disciplinar (materia o disciplina que se imparte), b) competencia pedagógica (metodología, comunicación, tutoría, evaluación, etc.), c) competencia tecnológica (aplicada al ámbito disciplinar, al pedagógico y a la investigación), y d) competencia de investigación e innovación (aplicada a su vez, a lo disciplinar, a lo pedagógico y a lo tecnológico). En suma, toda una base de reflexión para la capacitación de docentes. 
Palabras clave: educación a distancia; saber científico, teórico, técnico y práctico; competencias disciplinares, pedagógicas, tecnológicas y de investigación.

\begin{abstract}
This work intends to articulate the study and scientific research, in order to generate theories consistent with the educational practice and technical procedures put into action for the purpose of greater consistency, quality and, therefore, credibility to non-face-to-face educational formats. Thus, we discuss a brief delimitation on the different parameters of knowledge in the educational field, to propose afterwards, by looking into their relationships, the commitments and skills that teachers of this modality -above all those in higher educational levels- should integrate into their professional knowledge. Consequently, the four most relevant aspects of knowledge in education -scientific, theoretical, technical and practical knowledge, and the intersections between them- are described leading to the commitments and skills of higher education teachers who work in non-face-to-face educational formats. We conclude that these commitments would be of a professional, ethical, formative, collaborative and management nature. In this framework, the areas of teaching skills covering these four aspects would be included: a) subject competence (subject or discipline taught), b) pedagogical competence (methodology, communication, tutoring, evaluation, etc.), c) technological competence (applied to the subject, pedagogy and research), and d) research and innovation competence (applied, in turn, to the epistemological, pedagogical and technological fields). In sum, a holistic reflection on teacher training.
\end{abstract}

Keywords: distance education; scientific, theoretical, technical and practical knowledge; epistemological, pedagogical, technological and research skills.

En el ámbito de los saberes sobre educación en general y sobre educación a distancia en sus diferentes formulaciones, en particular, parecería lo más coherente el que existiese la máxima concordancia entre los diferentes tipos de conocimiento y de saberes cuyo objeto es la educación (Coll, Onrubia y Mauri, 2008). Sin embargo, no es lo más habitual encontrar relaciones sólidas, por ejemplo, entre los saberes científicos y teóricos sobre educación a distancia y los saberes ligados a la acción, sean prácticos o técnicos, en los desarrollos educativos en esta modalidad (Clará y Mauri, 2010). Un esfuerzo que tratase de articular el estudio, esto es, la investigación científica que genere teorías consistentes, con la práctica educativa y los procedimientos técnicos puestos en acción, dotaría de mayor consistencia, calidad y, por tanto, credibilidad a los formatos educativos no presenciales (figura 1). 
Figura 1. Dirección de los saberes sobre Educación
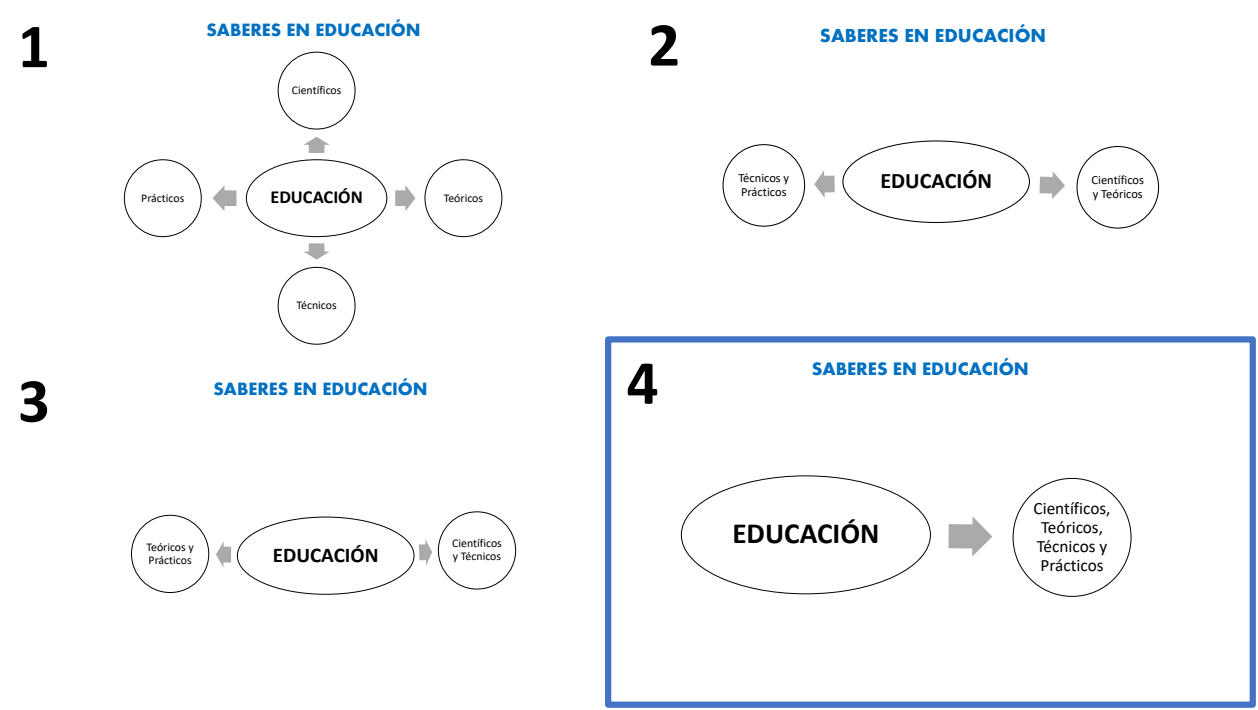

En el cuadrante 1 de esta figura, cada uno de los saberes apunta en sentido diferente. En el cuadrante 2 parecería que existe alguna cercanía mayor entre los saberes científico y teórico, por una parte, y los saberes técnicos y prácticos, por otra. El cuadrante 3 nos aproxima a otra forma de ver o entender estos conocimientos, sin problemas para relacionar lo científico y teórico en un enfoque y los teórico y práctico en otro. Y, finalmente, a la concordancia que proponemos entre los cuatro ámbitos de saberes, todos apuntando en dirección concordante.

Debemos avanzar que, en cuanto a resultados medibles, cifrados en el rendimiento y en el logro de aprendizajes, no parece que la educación a distancia deba "sonrojarse" al comparar los logros académicos de sus estudiantes con los de la educación presencial (García Aretio, 2017). Sin embargo, en cuanto a la relación, concordancia y coherencia de las propuestas teóricas con respecto a las realizaciones prácticas, tanto en los formatos presenciales como a distancia, en el entorno de los saberes pedagógicos no se constata optimismo al respecto, dado que sigue existiendo distancia entre lo que se estudia e investiga, lo que se practica y los procedimientos técnicos puestos en juego. Con ello, quisiéramos reforzar nuestro convencimiento de que, si existiese esa concordancia entre los distintos saberes, parecería que la calidad de los procesos educativos podría incrementarse.

En el presente trabajo abogamos por una formación de docentes, expertos e investigadores en el campo de la educación a distancia, hoy habitualmente sustentada en soportes digitales, que trate de aproximar al máximo los esfuerzos ligados a la investigación y a la innovación en esta modalidad con la práctica docente. En el caso de una investigación, una innovación, cuyos resultados no tuviesen incidencia 
alguna en la calidad de la acción educativa, se conformarían como una inversión de recursos económicos y humanos absolutamente baldíos.

En la búsqueda de esta concordancia y relación, ofrecemos en primer lugar una breve delimitación sobre los diferentes ámbitos del conocimiento y de los saberes en el campo educativo para posteriormente, buscando esa relación, proponer los compromisos y las competencias que un docente de esta modalidad, preferentemente de nivel educativo superior, debería integrar en su corpus de conocimientos profesionales. Resulta obvio que no sería exigible a cualquier docente de educación a distancia acercarse a todo este tipo de saberes en profundidad; eso lo dejaríamos para los estudiosos e investigadores que tomasen, precisamente como objeto de estudio, a la educación a distancia. Los docentes deberán buscar esas concordancias entre los resultados de las investigaciones y su acción educadora. Estimamos que el trabajo aporta elementos para la reflexión, enfocada a la formación de docentes y expertos en educación a distancia, que hoy se sustenta fundamentalmente en soportes digitales.

\section{LOS SABERES EN EDUCACIÓN A DISTANCIA}

Son diversas las formas en que quienes trabajamos como docentes, diseñadores, responsables institucionales, técnicos, estudiosos, investigadores, etc., de la educación a distancia, tenemos para aproximarnos a su conocimiento o para desenvolver las competencias requeridas en cada caso. Nos estamos refiriendo a las diferentes formas de aproximación al saber sobre la educación a distancia en sus múltiples manifestaciones, dimensiones y propuestas con que esta modalidad se nos presenta hoy y se nos ha presentado a lo largo de las últimas décadas. Proponemos las que entendemos como más destacadas formas de este saber: científico, teórico, práctico y técnico (Medina, 1981). Las significamos en la figura 2 visualizando como la intersección entre los cuatro ámbitos sería la ideal. 
Figura 2. Saberes pedagógicos y en educación a distancia

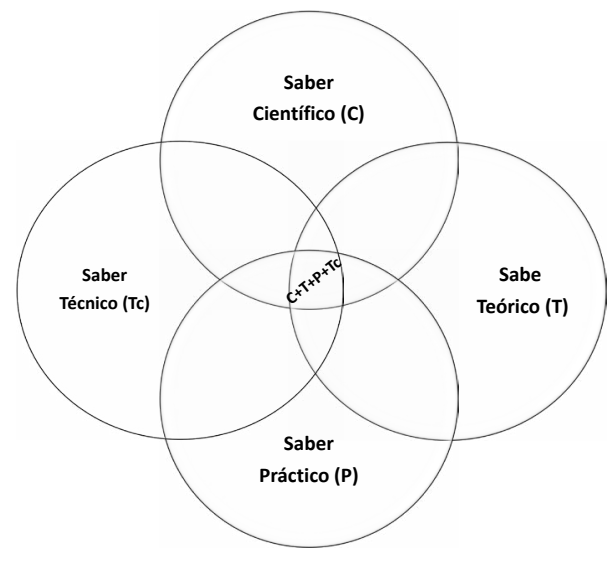

\section{El saber científico}

Esta forma de acercarnos al saber sobre educación sería en extremo demostrativa, necesaria, rigurosamente lógica y, en fin, deducida a partir de los primeros principios evidentes por sí mismos. Claro que, con estas características, ese saber científico quedaría muy reducido a las ciencias más formales, a las ciencias "duras”. A esas características propias de las primeras concepciones de saber científico, poco a poco se le fueron agregando otras tales como la inducción, las otras formas de demostración, no solo lógica, sino por verificación, falsación, testabilidad..., la ampliación, en fin, del concepto de ciencia hasta incluir saberes tradicionalmente adscritos a otros tipos de reflexión, tales como las ciencias sociales y humanas (Medina, 1981). De otra manera el saber científico en torno a la educación no existiría y, por ende, tampoco el referido a la educación a distancia.

No cabe duda de que nos encontraríamos ante la forma de conocer más garantista de resolver exitosamente los problemas que pudieran presentársenos en el campo de nuestro interés. Si los enunciados que pudiéramos afirmar sobre el campo que nos ocupa tienen la posibilidad de comprobarse, llegaríamos al pretendido asentimiento generalizado que se trataría de buscar. La ciencia pedagógica trata de elaborar sistemáticamente sus propios principios, ideas y conceptos en torno a su objeto de estudio, la educación, mediante la pertinente metodología de investigación, y pretende resolver los problemas teóricos y tecnológicos que le son propios. Y éste debería ser empeño de los estudiosos e investigadores de la educación a distancia: la reflexión, comprensión, interpretación, explicación, descripción, predicción, descubrimiento, justificación y prescripción de los múltiples hechos, situaciones y acciones educativas que sucedieron, ocurren o pueden producirse en este campo (Medina, 1981; Medina, Rodríguez y García-Aretio, 1992). 
Concebimos la pedagogía como la ciencia que aporta fundamentación teórica, tecnológica y axiológica, dirigida a explicar, interpretar, decidir y ordenar la práctica de la educación. Así, en el área que ahora nos importa y partiendo de que la educación a distancia ante todo es educación, nuestro reto debe ir encaminado a fundamentar, con el fin de poder explicar, interpretar, decidir y ordenar la práctica en los formatos educativos no presenciales.

Abogamos, por tanto, en educación a distancia, sin menospreciar, ni prescindir de las otras formas de conocer a las que ahora aludiremos, por unos conocimientos relacionados unos con otros de forma coherente, generalizables, con suficiente grado de objetividad y contrastados mediante métodos de verificación fiables.

\section{El saber teórico}

El saber teórico está muy ligado al saber científico, pero también al práctico, aunque la teoría en su concepción originaria hace mención al simple, o no tan simple, acto de ver, observar o contemplar. Es decir, en aquella concepción, limitarse a la observación sin que la misma se contamine con la actividad práctica. Sería algo así como el conocimiento por el conocimiento sin afán de aplicación posterior. Saber teórico sobre educación a distancia sería, simplemente, conocimiento de la educación a distancia. Pero ese conocimiento no es estático y para siempre, sino que es provisional, evoluciona y crece (Popper, 1962). Esa evolución, según los momentos, genera a su vez enfoques teóricos que pueden ser diferentes entre sí al exigir que, tras esa observación, se supiera describir y explicar esa realidad cuidando de relacionar los hechos y fenómenos de forma debidamente articulados. Y, si hablamos de evolución en el campo de la educación a distancia, los cambios han sido patentes y, en consecuencia, también los enfoques teóricos.

Aunque los saberes teóricos y prácticos se han venido considerando como opuestos, deberíamos entender que la teoría, tal y como se concibe hoy (alejada de su significado primigenio), sólo tendría sentido si se llega a convertir en un conocimiento instrumental al servicio de esa práctica, al ofrecer el conocimiento preciso para explicar las causas, cuestión que también estaría ligada al saber técnico. Así podríamos entender que teoría y práctica son saberes recíprocos, que deben estar integrados (Medina, 1981).

Actualmente la teoría amplía su cometido, yendo más allá de la pura contemplación, descripción o explicación dado que también desde la teoría se pueden abordar fenómenos dinámicos y procesos, como es el caso de la educación en general y de la educación a distancia en particular. Ya en otro lugar, en el que recogíamos ideas nuestras de hace más de dos décadas, postulábamos el sentido de una teoría de la educación a distancia como la construcción científica que consiste en la sistematización de las leyes, ideas, principios y normas, con objeto de describir, explicar, comprender y predecir el fenómeno educativo en la modalidad a distancia y regular la intervención pedagógica en este ámbito (García Aretio, 2001). Y en 
aquellos casos en que esa teoría no nos proporcionase reglas que puedan aplicarse para predecir y regular la intervención, es probable que nos facilite el lenguaje con el que construir descripciones más ajustadas (Schön, 1998).

Este conocimiento teórico así entendido estaría íntimamente ligado con los otros saberes. En todos los campos del saber se reconoce la importancia de los fundamentos teóricos para un desarrollo sólido de las realizaciones prácticas. No se puede avanzar, en nuestro caso en educación a distancia, si nos limitamos a mostrar sólo experiencias sobre qué es o cómo se hace o utiliza acá o allá una herramienta, un curso, una experiencia. Para describir una realidad y, quizás, para explicarla, no podemos limitarnos a mostrar datos descriptivos, perfiles, resultados sobre la experiencia, etc., aunque todo eso sea positivo y en muchos casos necesario. Han de hacerse propuestas teóricas explícitas que puedan discutirse y vayan ampliando su grado de consenso científico (Medina, Rodríguez y García-Aretio, 1992), y así reforzaríamos la calidad y los avances en los nuevos sistemas de enseñanza y aprendizaje digital. Han de comprenderse los diferentes modelos en los que se muestran estas diversas formas de enseñar y aprender.

\section{El saber práctico}

Resulta evidente que la pedagogía es una ciencia teórica y práctica a la vez, especulativa y normativa. Tal y como señalaba Hubert (1977), la pedagogía no es exclusivamente ni ciencia, ni técnica, ni filosofía, ni arte, sino todo ello a la vez y ordenado según articulaciones lógicas. Así, hablaríamos de una práctica apoyada en un saber científico y en una habilidad técnica que la facilita, y cimentada en unas ideas de valor que dan sentido de finalidad a la acción educadora (Medina, Rodríguez y García Aretio, 1992). Parece evidente, llegados aquí, que la teoría sin práctica difícilmente es posible, si es que no debe excluirse completamente. Sin embargo, la práctica sin teoría es posible, aunque es básicamente problemática y peligrosa en el educador profesional (Nassif, 1985).

Estaríamos hablando del saber basado en la experiencia, saber experimentado o saber espontáneo. Sería el saber propio del práctico, del educador a distancia que, a base de ensayo y error, por ejercicio, por hábito, sabe cómo actuar en las diferentes ocasiones que le brinda esta forma de educar. Es ésta una de las maneras más usuales de saber dentro de este ámbito. Se refiere a aquella intervención que está guiada por el sentido común, por los hábitos de los propios agentes, por la experiencia, la tradición, las creencias, las teorías implícitas... Se trata de una actuación que no se fundamenta en ninguna reflexión crítica consciente, ni en fundamentación científica alguna, sino únicamente en lo que es propio de esa forma de educar/enseñar según quienes la vienen practicando, por lo que, en consecuencia, se transmite de forma no sistemática (Medina, 1981).

En este sentido, la mayoría de los modelos y pautas de conducta de todos y cada uno de los agentes implicados se ponen en acción porque es lo asumido por la 
generalidad, sin llevarse a cabo ninguna reflexión previa sobre la adecuación o no de los mismos. No se da sistematización ni planificación intencional expresa, sino que por medio de la observación y de la experiencia se van asumiendo aprendizajes a partir de los diversos modelos y propuestas conocidos que nos rodean (Medina, 1981). Se trataría, en definitiva, de un saber hacer que se sostiene por uso, quizás rutina, e inculcación de hábitos.

Este saber sobre la educación a distancia sería un saber directo, como decimos, carente de reflexión o consciencia y, consecuentemente, desprovisto de argumentos que muestren el por qué se actúa así y no de otra manera. Se trataría de aplicar aquello que vi hacer, aquello que intuyo o aquello que vengo haciendo con no malos resultados, pero sin razones ni tecnológicas ni científicas de por qué hago lo que hago, aunque a eso que hago le aplique el sentido común. Parece que es a través de este saber mediante el que se educa de forma mayoritaria, no solo en educación a distancia, también en los sistemas más convencionales. Y en lo que nos ocupa en este espacio, con más probabilidad, la realidad quizás sea porque el conocimiento de la educación a distancia de carácter más fiable, científico, en definitiva, está aún "en pañales" y el poco que existe está escasamente asimilado y divulgado. Amén del convencimiento que muchos tienen de que ese tipo de conocimiento sirve para poco dado que lo que hacen, basado en la experiencia, funciona bien.

Naturalmente, no despreciamos, ni mucho menos, este tipo de saber. Es más, estamos convencidos de su valor y de la necesidad de que quienes no tienen experiencia realicen inmersión en situaciones prácticas exitosas que otros desarrollan. Además, no nos cabe la menor duda de que el práctico exitoso con sus aplicaciones relativas a la educación a distancia está aplicando, aún sin percatarse de ello, principios, saberes... experimentados, recogidos del ejercicio, de la práctica pero que tienen su fundamento teórico y su base tecnológica o científica. En todo caso, existen propuestas y corrientes que abogan por reconocer al profesor como quien debe generar su propia teoría respecto a casos concretos, más que aplicar simplemente teorías generales propuestas por otros.

\section{El saber técnico}

En esta ocasión nos estaríamos refiriendo al saber hacer, pero en este caso saber hacer la educación a distancia conociendo el por qué, la causa por la que se hace lo que se hace, las razones de por qué la actividad que se pone en práctica tiene éxito o puede tenerlo. En este saber, la teoría y la práctica confluyen. Incluso diríamos que lo sustantivo es más el saber que el hacer. La técnica es siempre medio, un instrumento adecuado para el desarrollo de una acción que implica habilidad, destreza..., además de la utilización de instrumentos.

Estaríamos hablando de la tecnología, pero entendida en este caso como la reflexión sistemática y procesual sobre el saber técnico, y no tanto, como habitualmente se hace, como cacharrería, herramientas o instrumentos más o menos 
sofisticada, como artefactos y artilugios. Desde este enfoque, un buen tecnólogo de la educación a distancia sería aquel, no que usa bien el hard y el soft, sino que actúa por razones bien fundamentadas; su especialidad serían las acciones racionales y su finalidad primordial el conseguir los resultados pretendidos con la mayor eficacia y eficiencia. Esa sería la base del proceder técnico, algo así como lo opuesto al proceder artesano. Las formas de actuar del artesano, por definición, no son científicas, actúa por tradición, por experiencia práctica, y por ello se puede dudar de su éxito porque la objetividad estaría ausente.

Sin embargo, no deberíamos confundir esta forma de entender la tecnología con la ciencia. Mientras que la ciencia busca leyes e interpreta hechos, la tecnología, que depende de aquella, persigue establecer normas e intervenir en la realidad para que suceda aquello que se pretende. En realidad, mientras el científico centra todo su esfuerzo en profundizar en el conocimiento, el técnico busca ese saber para hacer, en función de unos objetivos que, convenientemente ordenados, guían hacia un resultado previamente establecido. El saber técnico nos aportaría las bases de un modo eficaz de realizar la educación o, en un sentido más subjetivo, la habilidad o hábito que, siguiendo ciertas normas, va orientada a promover las acciones más convenientes en el proceder educativo (Medina, 1981; Medina, Rodríguez y GarcíaAretio, 1992).

Desde esta perspectiva sobraría hablar de tecnología cuando nos referimos sólo al ámbito de aplicación de las máquinas industriales, de la informática, de la telemática..., que no son otra cosa que las herramientas y las técnicas de que se vale la inteligencia para el logro de determinados fines. Por otra parte, también somos conscientes de que tanto la tecnología como la ciencia son conocimientos provisionales, como ya recordaba Popper (1962), en constante progreso mediante el proceso de comprobación, verificación y revisión tanto de los contenidos como de sus aplicaciones. Concordantemente, en educación a distancia se trataría de optimizar todas las variables que confluyeran en un determinado proyecto de acción formativa, planteándolas de forma estructurada, sistematizada, secuenciada y en complejidad creciente.

Es cierto que se ataca a este tipo de saber por su carácter eficientista al aplicar al hacer educativo los procedimientos de rentabilidad industrial o económica. Se buscan resultados, aplicación de técnicas con la idea de alcanzar los mejores productos de forma rápida y al menor coste posible, importando menos qué sea la educación a distancia y cómo mejorarla. Se haría difícil, con esta forma de proceder, el encontrar caminos diferentes que lleven a resultados distintos. El camino y los procedimientos, según este saber y entender tecnológico, tenderían a estar marcados y ser más unívocos. Estas normas con estructura uniforme preestablecida conformarían la aplicación extrema del paradigma tecnológico, más propiamente llamado en este caso tecnicista. El peligro es real habida cuenta de lo cómodo que resulta aceptar decisiones ajenas, perfectamente formalizadas, sin acudir a la crítica de éstas.

Este paradigma, así considerado, supondría una imposición de personas ajenas al proceso tanto para el alumno como para el docente, ejecutor de una 
acción planificada, previsiblemente elaborada y controlada desde fuera del ámbito propiamente del educador y del educando. Negar la flexibilidad a los procesos pedagógicos sería tratar a los educandos como autómatas sujetos a estímulos ante los que habrían de reaccionar de manera uniforme cuando, en realidad, una misma norma técnica aplicada simultáneamente a distintos sujetos provoca reacciones diferentes y asimilaciones no idénticas en los mismos. Y esto suele adivinarse en determinados programas de educación a distancia en formatos digitales.

Sin embargo, frente a estos planteamientos extremos, pensamos que los esquemas de acción de los que, gracias a la tecnología, un docente puede disponer hoy en los sistemas a distancia, mejoran la intervención educativa porque han sido elaborados con base en fundamentos científicos, con el fin de validar la práctica educativa.

El paradigma tecnológico sigue presentando una gran influencia en las diferentes propuestas educativas. Pensamos que mediante este saber se pretende la reflexión, elaboración, explicación y posterior aplicación de elementos y procesos propios de la intervención educativa. Esto es, racionalizar, sistematizar los medios y procedimientos y hacer eficaces las decisiones pedagógicas que tratan de responder a los problemas reales de esta modalidad educativa; generar técnicas de acción educativa partiendo del progreso de la ciencia pedagógica que, no se olvide, al igual que la técnica que de ella se deriva es de carácter provisional. Así se hace tecnología en educación.

Resulta lógico que este saber tecnológico exija a los educadores a distancia, más conocimientos científicos y técnicos sobre esta forma de educar/enseñar, más reflexión crítica sobre su propia práctica, en definitiva, más competencia profesional y esfuerzo que les aleje de actuaciones precientíficas y espontáneas. No se trata de aplicar pasivamente un repertorio de técnicas, sino considerar los contextos, las situaciones, los condicionamientos... decisiones que conducirán al logro de los fines establecidos, teniendo en cuenta, a la vez, las características del sujeto al que va dirigida esa acción y su contexto. La decisión habrá de fundamentarse científicamente con el fin de elegir la mejor opción que se adecue a ese caso único y singular. Exigiría de la intervención flexible, adecuada y planificada de acuerdo con una serie de variables que inciden en el desarrollo del proceso educativo.

Así, las normas técnicas, entendemos que, para un educador a distancia serían de obligado conocimiento, pero no como propuesta encorsetada de acción y de aplicación indiscriminada y uniforme. La conducta humana, las características del individuo y grupo, las circunstancias en que acaece la acción educativa, el entorno físico o virtual, son elementos que indiscutiblemente han de considerarse siempre en cualquier acción educativa, además de que debería incentivarse el componente creativo de cada sujeto y de cada educador.

De esta manera, esas normas generadas desde el proceder tecnológico pueden valer, y mucho, para una acción planificada pero realimentada constantemente por esas variables antes señaladas y por otras que pudieran interferir en el proceso. Así estaríamos ante una forma de concebir la técnica aplicada a la educación que sea 
abierta, flexible y positiva. Por todo ello, la actividad educativa en este campo ha de ser desempeñada por profesionales, tecnólogos que actúen científicamente, que comprendan los problemas educativos y el repertorio de conocimientos científicos que sobre el particular existen, los casos similares ya resueltos y las técnicas que permiten abordarlos.

Podríamos resumir con Henz (1968), que la pedagogía como ciencia teóricopráctica hace referencia simultáneamente a la teoría, al conocimiento científico del hecho educativo y a la aplicación práctica de ese conocimiento, al análisis científico de aquel hecho y al conjunto de normas derivadas de ese análisis (técnica) que guían y orientan el proceso educativo. Más allá de que en algunas circunstancias el sujeto sea dueño de uno solo de esos saberes, las diferentes combinaciones entre ellos que, tal como hemos tratado de exponer, en unos casos son más frecuentes que en otros y serían las que mostramos en la figura 3. Obviamente, apostamos por la concordancia de los cuatro saberes tal y como precisábamos en las intersecciones de la figura 2 $(\mathrm{C}+\mathrm{T}+\mathrm{Tc}+\mathrm{P})$.

Figura 3. Intersecciones entre los cuatro saberes
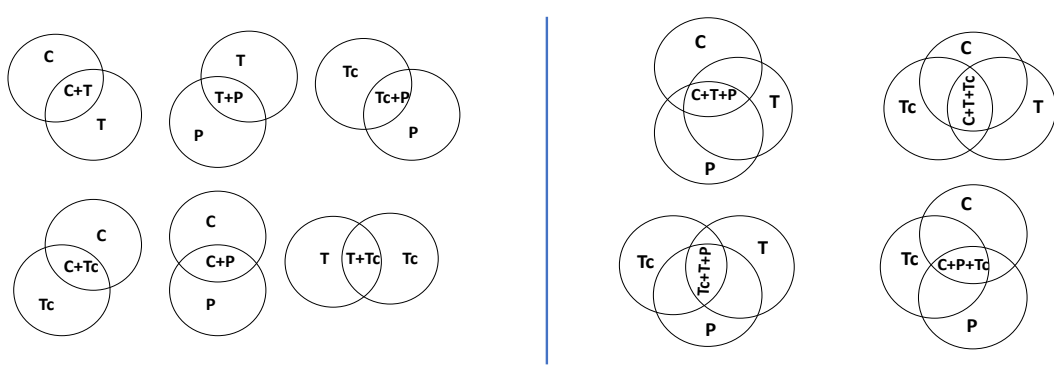

\section{CONOCIMIENTOS, COMPROMISOS Y COMPETENCIAS EN LA DOCENCIA A DISTANCIA}

Pues bien, realizado el repaso de esas modalidades de conocimientos relacionados con la educación en general y con la educación a distancia en particular, trataremos de concretar en la realidad de los entornos educativos a distancia. Es decir, nos importa el docente de educación a distancia en cualesquiera de los campos del saber, partiendo de la base de que la profesión del docente es de las más comprometedoras dada la responsabilidad personal y social que se asume. Parece que ante ese reto un profesional docente no se puede improvisar. Un profesor que está preparado para ejercer su tarea quiere decirse que trata de poner en acto una serie de conocimientos, compromisos y competencias que no son otra cosa que las obligaciones contraídas con la sociedad, con la institución y con sus estudiantes basadas en las propuestas avanzadas en el apartado anterior. 
Aunque la propuesta explicitada en torno a los saberes pedagógicos en educación a distancia ha obviado algo imprescindible en el docente a distancia, en cualquier docente, el saber correspondiente a la disciplina que imparte y que, en este trabajo, lo debemos dar por supuesto. Más allá de que puedan existir didácticas específicas relativas a la disciplina o materia objeto de docencia.

La propuesta que vamos a realizar referida a los conocimientos y competencias bien podría servir tanto para docentes presenciales como a distancia. Sin embargo, existen una serie de compromisos que resultan más propios de aquellos que realizan su actividad en entornos virtuales. En concreto, nos importa aquí destacar la relevancia de integrar los conocimientos y competencias técnicas y tecnológicas en los procesos de enseñanza, sin olvidar los otros. De ahí la necesidad aún más imperiosa de una formación docente de calidad (Rodríguez, Prieto y Vázquez, 2014)

Vamos a partir de la estructura gráfica del modelo TPACK (Technological, Pedagogical, Content Knowledge) de Mishra y Koehler (2006) y Koehler y Mishra (2008), con raíces en el enfoque del conocimiento pedagógico del contenido de Schulman (1986), que puede orientar al profesorado para esa integración de la tecnología en los procesos educativos. Estos autores tratan de enfatizar la interacción de tres tipos de conocimientos: a) pedagógicos sobre la manera de ejercer docencia de calidad, b) disciplinares sobre los contenidos de la disciplina o materia a impartir, y c) tecnológicos sobre la forma de aplicar las tecnologías, de acuerdo con los otros dos conocimientos. Si esos tres ámbitos se integran adecuadamente se producirá una mejora de la calidad, según indican los autores.

Pero no bastaría con tener claros esos tres ángulos de forma aislada, sino que debe asimilarlos en forma de interacción entre los mismos, dando así lugar a las siete zonas diferentes que nos propone el modelo (Cabero, 2014a), lo que nos exigirá a los docentes diferentes tipos de competencias específicas (Cejas, Navío y Barroso, 2016). Y todo ello considerado dentro de un contexto socioinstitucional que siempre condiciona (figura 4).

- Conocimiento pedagógico (PK): Sobre pedagogía, didáctica y métodos de enseñanza.

- Conocimiento disciplinar (CK): Sobre la materia o disciplina asignada para la docencia.

- Conocimiento tecnológico (TK): Sobre las tecnologías actuales.

- Conocimiento pedagógico disciplinar (PCK): Didáctica de la materia, conocimiento de la disciplina y de cómo enseñarla.

- Conocimiento tecnológico disciplinar (TCK): Tecnologías más apropiadas para enseñar una materia concreta.

- Conocimiento tecnológico pedagógico (TPK): Las tecnologías en la educación. Usos pedagógicos de las TIC.

- Conocimiento tecnológico, pedagógico y disciplinar (TPACK): Integración de todos los conocimientos anteriormente señalados. 
Figura 4. Modelo TPACK sobre conocimientos pedagógicos, tecnológicos y disciplinares (Mishra y Koehler, 2006)

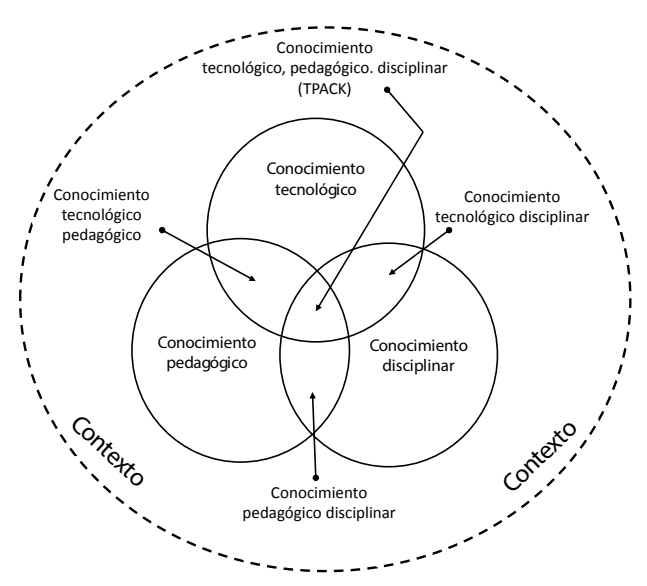

Este modelo ha sido estudiado y aplicado en muy diversas áreas curriculares (Deng, Sing, So, Qian y Chen, 2017; Hu, Walker y Hsiao, 2013), así como en diferentes niveles de formación del profesorado (Cabero-Almenara, Roig-Vila y MengualAndrés, 2017; Rienties et al., 2013), con resultados desiguales.

Sobre esta base gráfica, hemos construido nuestro esquema completando áreas que nos parecen relevantes dentro de los compromisos que ha de poner en juego un docente de hoy, sobre todo, si labora en un entorno de educación superior. De ahí la relevancia que le hemos asignado al compromiso investigador y de innovación, no contemplado en el modelo TPACK.

Además, tratamos de superar la esfera de las competencias para recalar en el ámbito de los compromisos, de las obligaciones contraídas que suponen algo así como la palabra dada, a la sociedad, a la institución y a cada uno de los estudiantes puestos en manos del docente, referida a que se cuenta con la formación requerida para el desempeño de tan relevante profesión. Esos compromisos derivan en conocimientos, competencias, actitudes, tareas, etc., que pueden sugerir pautas de acción para el buen docente. Compromisos que, de acuerdo con Bautista, Borges y Forés (2006), no deberían ser muy diferentes a los mostrados por los colegas de la enseñanza convencional, aunque sí los roles que cada cual habría de jugar. Aunque no ha de olvidarse la necesidad de enfatizar en la formación para la adquisición de competencias relativas a la adecuada integración de las TIC en un mundo digital (Krumsvik, 2012) y, más concretamente, en la docencia universitaria (Durán, Gutiérrez y Prendes, 2016; Cabero, 2014b; Tejada, 2014).

Por nuestra parte y como decimos, nos vamos a basar en una actualización de nuestra propuesta (García Aretio, 2014) que nos ayudará a explicitar esos compromisos que entendemos debe integrar el buen docente (figura 5), siempre 
teniendo en cuenta los saberes a los que hemos aludido en la primera parte de este trabajo. Deberemos partir del inevitable contexto socio-institucional en el que se encuentra inmersa nuestra labor docente: sociedad, institución, departamento, aula, taller, laboratorio... Habrán de considerarse todo tipo de condicionantes de carácter social, financiero, cultural, tecnológico y político de la zona e institución donde vaya a incardinarse por una parte la docencia y, por otra, el desempeño de la profesión (Bawane y Spector, 2009). Igualmente, no podrán obviarse los elementos propios de la planificación general de la institución o del programa de formación en cuestión. Esa planificación partirá de unas metas y objetivos institucionales que exigirán unos compromisos acordes con esos fines. La planificación concreta de la disciplina asignada que debería ser competencia de cada cual, también afectará a nuestro hacer educativo. Así, mirando desde el contexto socioinstitucional y la planificación, tanto institucional como disciplinar, la meta será que los estudiantes puestos a nuestro cargo alcancen finalmente aprendizajes valiosos y altos grados de satisfacción. Satisfacción que habría de medirse tanto a nivel interno como externo a la propia institución.

Figura 5. Compromisos y competencias del docente de calidad (García Aretio, 2014)

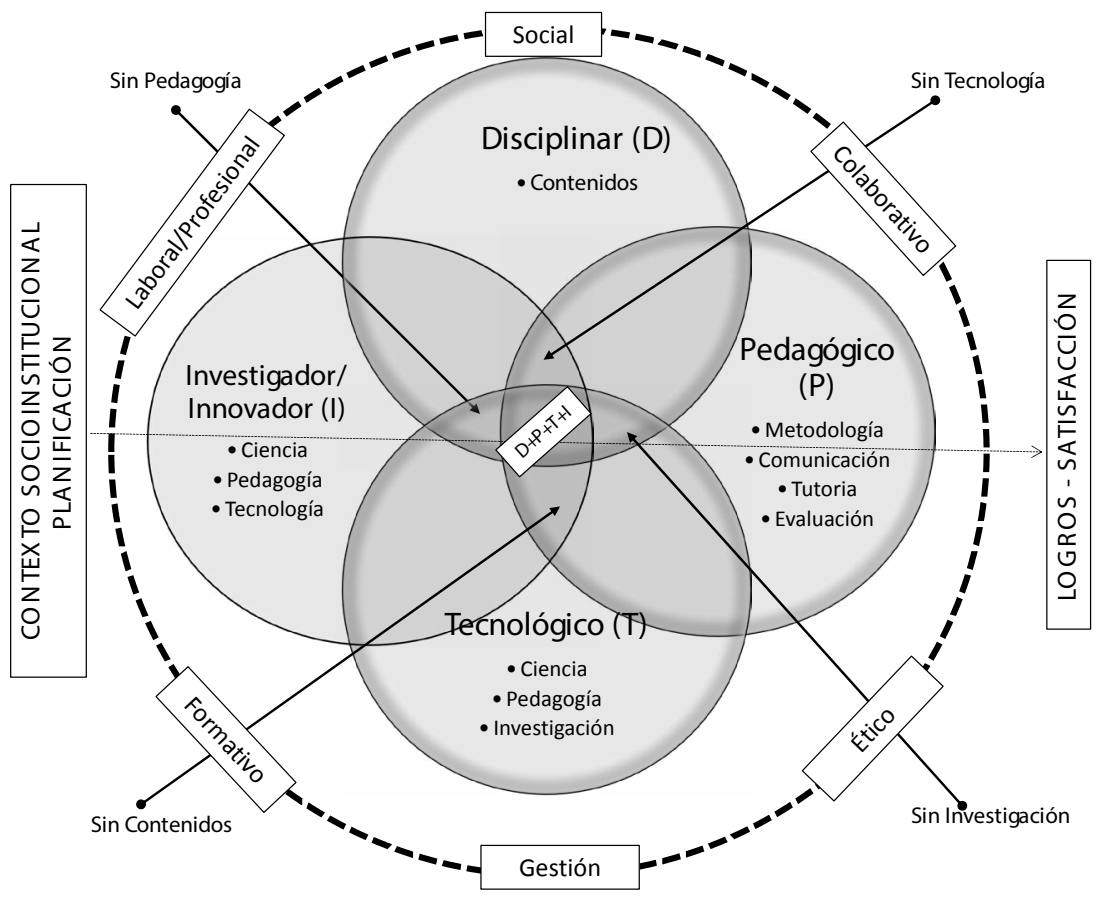


Señalada la dirección que ha de contemplar la buena práctica docente, observamos en el gráfico un contorno de compromisos irrenunciables a la responsabilidad de un buen profesor:

- Compromiso profesional-laboral. Debe saber lo que supone su misión y tarea como profesor o tutor, así como el ámbito propio y los límites de su actuación. Exige este compromiso cumplir las obligaciones contractuales asumidas, al más alto nivel. Ha de transformar la enseñanza en una profesión.

- Compromiso social. A través de los docentes, la sociedad hace posible que pueda satisfacerse un derecho fundamental, el de la educación. Se trata de una indeclinable responsabilidad de servicio a la sociedad.

- Compromiso ético. Deberá mostrar una incuestionable actitud ética en toda su actividad educadora. La ética profesional es la garantía última de nuestro trabajo. No puede educarse a cualquier precio, ni permitirse hablar de profesionalidad sin un compromiso deontológico serio en el trabajo.

- Compromiso formativo, de actualización. Hoy ninguna profesión puede satisfacer las demandas sociales y personales con la formación recibida en una preparación inicial más o menos lograda. Los avances sociales, tecnológicos, científicos..., están condicionando la marcha de toda profesión, de todo trabajo, por lo que va a exigir a cada uno la constante puesta al día. Saber anticiparse, generar nuevos conocimientos, profundizar en los procesos..., son elementos claves de la formación permanente de todo docente.

- Compromiso colaborativo. Compartir su pensamiento y acción docente con los otros miembros del equipo docente y otros colegas. Este compromiso habría de extenderse más allá del núcleo institucional participando en algunas de las numerosas redes diseñadas para la colaboración y el aprendizaje entre pares.

- Compromiso de gestión. Aunque no obligado, de acuerdo con la legislación universitaria española. La gestión del docente universitario se entiende como el compromiso personal que cada uno adquiera voluntariamente con la institución donde trabaja. Y en concreto, en una universidad, son numerosas y muy diversas las tareas de gestión que deberían desempeñar los docentes.

Si repasamos otros estudios, en concordancia con lo señalado, podrían destacarse como cinco roles profesionales del docente: el de especialista en su disciplina, el de docente y tutor de sus alumnos, el de investigador, el de profesional en su área (paralela a su actividad como docente) y el de gestor en alguna de las áreas (Cejas, Navío, y Barroso, (2016). Por su parte, Zabalza (2003) reflexiona sobre las competencias del docente universitario: planificar el proceso de enseñanza aprendizaje, preparar los contenidos, ofrecer explicaciones bien organizadas, utilizar las tecnologías, diseñar la metodología, relacionarse con los estudiantes, fortalecer el trabajo en equipo, desarrollar la tutoría, realizar la gestión académica, reflexionar sobre su práctica docente y evaluar. Sin embargo, bien es sabido que no todas esas funciones, roles 
o competencias, según se pretenda definirlos son equiparables en reconocimiento, incentivos y satisfacción personal (Galán, González y Román, 2012; Tomàs e Ion, 2008; Tomàs, Castro y Feixas, 2012). En concreto, los estudios señalados apuntan en la dirección de que los docentes de hoy, fundamentalmente los universitarios, miran de forma especial la investigación, relegando a un segundo o tercer nivel los otros compromisos. Nosotros entendemos que todos ellos, de una u otra forma, con mayor o menos énfasis, deben integrarse en la función docente.

De acuerdo con nuestro esquema adaptado (García Aretio, 2014), vamos a sintetizar esos perfiles, funciones o competencias del docente universitario, centrándolos en los cuatro que aparecen recogidos en los círculos correspondientes: disciplinar, pedagógico, tecnológico e investigador/innovador.

Una vez asumidos esos compromisos que conforman el contorno de nuestro gráfico y que podrían valer probablemente para otras profesiones, trataremos de concretar otros más específicos que apuntan directamente a esa docencia de calidad que venimos pretendiendo, específicamente para entornos de educación a distancia. Observamos cuatro grandes círculos que encierran saberes docentes inevitables (científicos, teóricos, técnicos y prácticos), sobre todo en contextos de educación superior.

- Compromiso disciplinar (contenidos científicos). Conocer bien, dominar, aquello que se ha de enseñar. Se trata de la competencia científica sobre la asignatura o campo disciplinar, que se traduce en: rigor académico, estar al día, mantener estándares profesionales, generar y validar ese conocimiento.

- Compromiso pedagógico/didáctico. En este apartado resulta imprescindible atenuar y suplir la ausencia física del docente, a través de una comunicación de calidad (Alamri y Tyler-Wood, 2017). Saber, en nuestro caso, cómo enseñar a distancia, de acuerdo con los recursos tecnológicos disponibles por parte del docente y de sus alumnos. Este compromiso comporta una serie de competencias (Llorente, Cabero y Barroso, 2015; Torra y otros, 2012; Mas, 2012; Zabalza, 2003):

- Competencia metodológica que supone el conocimiento de las diferentes vías para el aprendizaje, saber planificar y diseñar la correspondiente guía y la materia, conocer estrategias de desarrollo curricular (espacios, métodos, actividades...), diagnosticar alumnos y grupos, evaluar logros.

- Competencia comunicacional que supone cómo puede y debe relacionarse y tratar al que (a los que) aprende(n). Ello exige propiciar procesos multidireccionales de comunicación de manera eficaz y correcta, lo cual implica la recepción, interpretación, producción y transmisión de mensajes a través de canales y medios diferentes y de forma contextualizada a la situación de enseñanza-aprendizaje, así como ofrecer información y explicaciones comprensibles y bien organizadas con alumnos a nivel individual y grupal. 
- Competencia tutorial, ser tutor del proceso de aprendizaje del estudiante, y establecer las relaciones y la comunicación interpersonal que reclama la función: atención individual que potencie intereses y necesidades y aborde las dificultades.

- Compromiso tecnológico. Que exige el saber hacer, aplicando las normas y recursos más convenientes enfocados a logros de aprendizaje, integrando en el modelo los recursos tecnológicos institucionales y los de sus propios alumnos, con conciencia de las oportunidades e implicaciones del uso de las tecnologías en los procesos de enseñanza y aprendizaje, que nos obliga a conocer las aplicaciones de las TIC en el campo disciplinar, desde la perspectiva tanto de las fuentes documentales, como de la metodología de enseñanza. Tecnologías que habrán de integrarse tanto al ámbito disciplinar como al pedagógico y al investigador.

- Compromiso investigador e innovador. Compromiso exigido a cualquier docente y de manera más especial a los que laboran en ámbitos de educación superior. Investigación unida a la necesaria innovación, que suponen: reflexión sobre el área disciplinar y sobre su práctica docente; indagación pedagógica y apertura a las innovaciones metodológicas; creación y aplicación de nuevos conocimientos, perspectivas, metodologías y recursos en las diferentes dimensiones de la actividad docente, orientados a la mejora de la calidad del proceso de enseñanza-aprendizaje. Todo educador debe ser investigador de su propia práctica docente, lo que le va a ayudar a mejorar su tarea. La verdad es que el docente universitario generalmente se identifica bien con esta competencia, pero fundamentalmente centrada en la investigación en torno a la disciplina (Monereo y Domínguez, 2014).

Parece que con cada uno de esos cuatro círculos (compromisos) por sí mismo, aislados, estaríamos lejos de un docente de calidad. Contemplemos ahora brevemente las intersecciones de estos círculos de las que se derivan otras seis áreas algo más completas porque suponen la asunción de dos de esos cuatro compromisos (figura $6)$. 
Figura 6. Intersecciones duales de los cuatro compromisos básicos
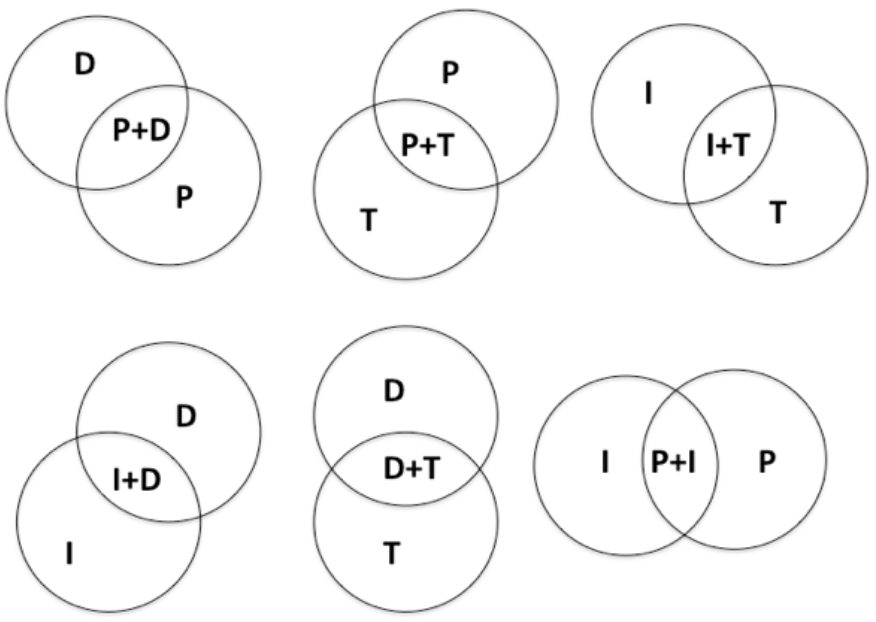

- Pedagógico-Disciplinar $(P+D)$. Sería un compromiso ligado al conocimiento de la didáctica de la materia o disciplina en cuestión. Una cosa es conocer la asignatura y otra saber cómo enseñarla. Se busca la integración del contenido de la disciplina con la pedagogía (Vergara y Cofré, 2014)

- Pedagógico-Tecnológico $(P+T)$. Serían saberes relacionado con el uso pedagógico de las tecnologías. ¿Qué tecnologías según objetivos, contenidos, métodos, actividades...?, ¿qué procedimientos y normas?, ¿qué estrategias de enseñanza con TIC?, ventajas e inconvenientes para cada caso...

- Investigador-Tecnológico $(I+T)$. Esta área quedaría reservada para todas las implicaciones y usos de las tecnologías en la investigación y para la investigación e innovación. Y al revés, podrá suponer la investigación e innovación sobre las propias tecnologías.

- Investigador-Disciplinar $(I+D)$. Parecería como ámbito fundamental en la universidad. Investigar sobre los contenidos disciplinares, construir el propio conocimiento sobre la disciplina motivo de docencia que es donde parece que los docentes universitarios se sienten más y mejor identificados (Monereo y Domínguez, 2014).

- Disciplinar-Tecnológico $(D+T)$. Qué tecnologías para qué disciplina concreta. En determinadas materias existen herramientas y recursos tecnológicos bien desarrollados para ser aplicados a determinados procesos. Aplicación de las tecnologías al ámbito disciplinar.

- Pedagógico-Investigador $(P+I)$. Ámbito propio de la investigación pedagógica. Investigar para mejorar diseños y métodos pedagógicos, procesos de evaluación, etc. 
Pero ¿y si contemplamos a docentes con tres de los cuatro compromisos bien asumidos? Estaríamos más cerca del ideal de docente. Contemplemos estas otras cuatro áreas en la intersección de tres círculos de compromisos (figura 7):

Figura 7. Asumiendo tres de los cuatro compromisos
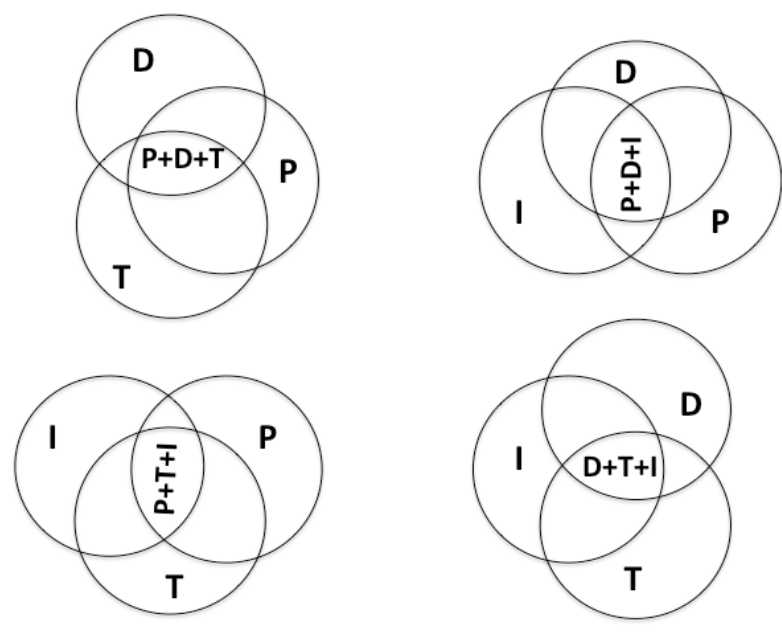

- Pedagógico-Disciplinar-Tecnológico $(P+D+T)$. Aunque defendemos la investigación-acción en todos los niveles, cierto que este ámbito en el que sólo falta la investigación podría ser válido para los niveles no universitarios.

- Pedagógico-Disciplinar-Investigador $(P+D+I)$. Se prescinde de la tecnología por lo que en esta sociedad digital estos docentes se nos presentarían con bastantes deficiencias.

- Pedagógico-Tecnológico-Investigador $(P+T+I)$. Sin dominar plenamente los contenidos disciplinares de la materia o disciplina, se estará abocado al fracaso, aunque se trabajen los otros compromisos.

- Disciplinar-Tecnológico-Investigador $(D+T+I)$. Ámbito de actuación muy propio de docentes universitarios que en buen número ignoran a la pedagogía como componente sustancial de una docencia de calidad.

Concluyendo: nos quedaría por considerar el área de ese docente ideal anhelado, el que integra plenamente los cuatro círculos, los cuatro compromisos. Sería la pequeña área central del gráfico de la figura $3(\mathrm{D}+\mathrm{P}+\mathrm{T}+\mathrm{I})$. Ese espacio supone integrar lo que el docente sabe sobre la materia que desea impartir, los métodos pedagógicos más adecuados a la situación concreta de los alumnos, la tecnología más apropiada para el caso y a todo ello sumado un interés por investigar, reflexionar 
sobre su práctica y sobre la propia disciplina, con el fin de innovar sobre la materia, sobre los procesos pedagógicos y sobre la propia tecnología.

Pensamos, en fin, que, por una parte, integrando los saberes pedagógicos y, por otra, diseñando los elementos concordantes con cada uno de los compromisos que entendemos debe asumir un docente, especialmente en el ámbito universitario, podrían conformarse acciones formativas para la capacitación de docentes y expertos en sistemas digitales de educación.

\section{REFERENCIAS}

Alamri, A., y Tyler-Wood, T. (2017). Factors Affecting Learners with Disabilities Instructor Interaction in Online Learning. Journal of Special Education Technology, 32(2), 59-69. https://doi. org/10.1177/0162643416681497

Bautista, G., Borges, F., y Forés, A. (2006). Didáctica universitaria en entornos virtuales de enseñanza-aprendizaje. Madrid: Narcea.

Bawane, J., y Spector, J. (2009). Prioritization of online instructor roles: Implications for competency-based teacher education programs. Distance Education, 30(3), 383-397.

Cabero, J. (2014a). La formación del profesorado en TIC: Modelo TPACK (conocimiento tecnológico, pedagógico y de contenido). Sevilla: Secretariado de Recursos Audiovisuales y Nuevas Tecnologías de la Universidad de Sevilla.

Cabero, J. (2014b). Formación del profesorado universitario en TIC. Aplicación del método Delphi para la selección de los contenidos formativos. Educación XX1, 17(1), 109-132. https:// doi.org/10.5944/educxx1.17.1.10707

Cabero-Almenara, J., Roig-Vila, R., y Mengual-Andrés, S. (2017). Conocimientos tecnológicos, pedagógicos y disciplinares de los futuros docentes según el modelo TPACK. Digital Education Review, 32, 73-84. Recuperado de http://revistes.ub.edu/index.php/der/ arti-cle/view/16981
Cejas, R., Navío, A., y Barroso, J. (2016). Las competencias del profesorado universitario desde el modelo TPACK (conocimiento tecnológico y pedagógico del contenido). Píxel-Bit. Revista de Medios y Educación, 49, 105-119. http:// dx.doi.org/10.12795/pixel- bit.2016. i49.07

Clarà, M., y Mauri, T. (2010). El Conocimiento práctico. Cuatro conceptualizaciones constructivistas de las relaciones entre conocimiento teórico y práctica educativa. Infancia y Aprendizaje, 33(2), 131-141.

Coll, C., Onrubia, J., y Mauri, T. (2008). Ayudar a aprender en contextos educativos: el ejercicio de la influencia educativa y el análisis de la enseñanza. Revista de Educación, 364, 33-70.

Deng, F., Sing, Ch., So, H-J., Qian, Y., y Chen, L. (2017). Examining the validity of the Technological Pedagogical Content Knowledge (TPACK) framework for preservice chemistry teachers. Australasian Journal of Educational Technology, 33(3), 1-14.

Durán, M., Gutiérrez, I., y Prendes, M. P. (2016). Análisis conceptual de modelos de competencia digital del profesorado universitario. RELATEC Revista Latinoamericana de Tecnología Educativa, 15(1), 97-114. https://doi. org/10.17398/1695288X.15.1.97

Galán, A., González, M. A., y Román, M. (2012). La irrupción del factor comunitario en el perfil del profesor universitario. Bordon, 64(3), 133-148. 
García Aretio, L. (2001). La educación a distancia, de la teoría a la práctica. Barcelona: Ariel.

García Aretio, L. (2014). Bases, mediaciones y futuro de la educación a distancia en la sociedad digital. Madrid: Síntesis.

García Aretio, L. (2017). Educación a distancia y virtual: calidad, disrupción, aprendizajes adaptativo y móvil. RIED. Revista Iberoamericana de Educación a Distancia, 2O(2), 9-25. https://doi. org/10.5944/ried.20.2.18737

Henz, H. (1968). Tratado de Pedagogía sistemática. Barcelona: Herder.

Hu, H-W., Walker, K., y Hsiao, W-Y. (2013). Developing elementary pre-service teachers' technological, pedagogical, and content knowledge for learning and teaching division of fractions. Inter-national Journal of Technology, Knowledge and Society, 9(2),185-204. https://doi.org/10.18848/1832-3669/ CGP/vogio2/56380

Hubert, R. (1977). Tratado de Pedagogía general. Buenos Aires: El Ateneo.

Koehler, M. J., y Mishra, P. (2008). Introducing technological pedagogical knowledge. In AACTE (Eds.), The handbook of technological pedagogical content knowledge for educators (pp. 3-28). New York: Routledge, Taylor \& Francis Group.

Krumsvik, R. J. (2012). Teacher educators' digital competence. Scandinavian Journal of Educational Research, 58(3), 269-280. https://doi.org/10.1080/00313 831.2012.726273

Llorente, M. C., Cabero, J., y Barroso, J. (2015). El papel del profesorado y alumnado en los nuevos entornos tecnológicos. En J. Cabero y J. Barroso (Eds.), Nuevos retos en tecnología educativa (217-236). Madrid: Síntesis.

Mas, O. (2012). Las competencias del docente universitario/: la percepción del alumno, de los expertos y del propio protagonista. REDU Revista de Docencia Universitaria, 10 (2), 299-318.

Medina, R, (1981). La diversidad de niveles de acceso al conocimiento de los fenómenos educativos. Aula abierta, 32.

Medina, R., Rodríguez, T., y García-Aretio, L. (1992). Teoría de la educación. Madrid: UNED.

Mishra, P., y Koehler, J. (2006). Technological Pedagogical Content Knowledge: A new framework for teacher knowledge. Teachers College Record, 108(6), 1017-1054. doi: 10.1111/j.14679620.2006.00684.x.

Monereo, C., y Domínguez, C. (2014). La identidad docente de los profesores universitarios competentes. Educación $X X 1, \quad 17(2), \quad 83-104 . \quad$ http://doi. org/10.5944/educxx1.17.2.11480

Nassif, R. (1985). Teoría de la educación. Madrid: Cincel.

Popper, K. (1962). La lógica de la investigación científica. Madrid: Tecnos.

Rienties, B., Brouwer, N., Carbonell, K. B., Townsend, D., Rozendal, A-P., Loo, J., Dekker, P. y Lygo-Baker, S. (2013). Online training of TPACK skills of higher education scholars: A crossinstitutional impactstudy. European Journal of Teacher Education, 36(4), 480-495. $\quad$ https://doi.org/10.10 80/02619768.2013.801073

Rodríguez, A., Prieto, M., y Vázquez, R. (2014). El uso de las TIC en la formación permanente del profesorado para la mejora de su práctica docente.Etic@ Net, I, http://dx.doi.org/10.12795/ pixelbit.2016.i49.07

Schön, D. A. (1998). El profesional reflexivo. Cómo piensan los profesionales cuando actúan. Barcelona: Ediciones Paidós Ibérica, S.A.

Schulman, L. S. (1986). Those who understand: knowledge growth in teaching. Educational Researcher, 15(2), 1-22. 
Tejada, J. (2014). Formando formadores: nuevos escenarios y competencias digitales docentes. En V. Marín (Coord.), El hoy y el mañana junto a las TIC. (94154). Sevilla: Universidad de Sevilla.

Tomas, M., Castro, D., y Feixas, M. (2012). Tensiones entre las funciones docente e investigadora del profesorado en la universidad. Revista de docencia universitaria. REDU, 1O(1), 343-367.

Torra, I., Corral, I., Pérez, M., Pagès, T., Valderrama, E., Màrquez, M., Sabaté, S., Solà, P., Hernàndez, C., Sangrà, A., Guàrdia, L., Estebanell, M., Patiño, J., González, À., Fandos, M., Ruiz, N., Iglesias, M., Tena, A., y Triadó, X. (2012). Identificación de competencias docentes que orienten el desarrollo de planes de formación dirigidos a profesorado universitario. REDU. Revista de Docencia Universitaria, 10(2), 21-56. https://doi. org/10.4995/redu.2012.6096

Vergara, C., y Cofré, H. (2014). Conocimiento Pedagógico del Contenido: ¿el paradigma perdido en la formación inicial y continua de profesores en Chile? Estudios Pedagógicos, XL, 323338. http://doi.org/10.4067/So71807052014000200019

Zabalza, M. A. (2003). Las competencias docentes del profesorado universitario. Calidad y desarrollo profesional. Madrid: Narcea. 